\title{
Antibiotic-resistant bacterial colonization increases the number of hospitalizations in patients after solid organ transplantation or with non-communicable diseases
}

\author{
Bożena Czarkowska-Pączek1,2,A,C-F, Elżbieta Wawiórko ${ }^{1, B, E, F}$, Grażyna Młynarczyk ${ }^{2, B, E, F}$, Leszek Paczek 3 3,A,C,E,F \\ ${ }^{1}$ Department of Clinical Nursing, Medical University of Warsaw, Poland \\ ${ }^{2}$ Chair and Department of Medical Microbiology, Medical University of Warsaw, Poland \\ ${ }^{3}$ Department of Immunology, Transplantology and Internal Diseases, Medical University of Warsaw, Poland \\ A - research concept and design; B - collection and/or assembly of data; C - data analysis and interpretation; \\ $D$ - writing the article; $E$ - critical revision of the article; $F$ - final approval of the article
}

\section{Address for correspondence}

Bożena Czarkowska-Pączek

E-mail:bozena.czarkowska-paczek@wum.edu.pl

\section{Funding sources}

None declared

Conflict of interest

None declared

Received on April 18, 2019

Reviewed on May 22, 2019

Accepted on January 2, 2020

Published online on March 24, 2020

Cite as

Czarkowska-Pączek B, Wawiórko E, Młynarczyk G, Paczek L. Antibiotic resistant bacterial colonization increases the number of hospitalizations in patients after solid organ transplantation or with non-communicable diseases. Adv Clin Exp Med. 2020;29(3):307-312. doi:10.17219/acem/116068

DOI

10.17219/acem/116068

Copyright

Copyright by Author(s)

This is an article distributed under the terms of the

Creative Commons Attribution 3.0 Unported (CC BY 3.0)

(https://creativecommons.org/licenses/by/3.0/)

\begin{abstract}
Background. Healthcare-associated infections could affect the rate of morbidity, mortality and postdischarge hospitalization among patients. They are also dangerous to healthcare professionals and generate significant cost to the healthcare system.
\end{abstract}

Objectives. The aim of this study was to evaluate the occurrence rate of colonization with various antibioticresistant (AR) bacteria among patients admitted to the Department of Immunology, Transplantology and Internal Diseases.

Material and methods. The study used retrospective analysis of patients $(n=280)$ with no clinical signs of infection admitted into the department between November 2015 and May 2017. The observational period lasted until January 2019. Collected data included sex, age at admission, location directly prior to current hospitalization, and medical history. Nasal and rectal swabs were collected, and stool and urine samples were obtained on the day of admission. Specimens were cultured according to standard microbiological procedures. In all cases, the appropriate bioMerieux (Marcy-l'Étoile, France) media were used. Isolates were identified using mass spectrometer (Vitek MS; bioMerieux).

Results. One-hundred ninety-one (68.2\%) of patients were colonized with AR bacteria. The incidence of colonization was not influenced by age or sex. The risk of colonization was associated with admission from another hospital and history of kidney transplantation ( $p=0.0136$ and $p<0.001$, respectively). The number of hospitalizations during the whole observational period was higher in the group of colonized patients compared to non-colonized ( $2.76 \pm 2.4$ vs $2.07 \pm 1.68, p=0.0099$ ). The number of hospitalizations correlated positively with the number of positive cultures obtained from the same patients ( $r h 0=0.18, p=0.0274$ ).

Conclusions. The rate of colonization at admission to the ward could be high, depending on previous hospitalization and medical history. Colonization significantly increased post-discharge hospitalization rate.

Key words: hospitalization rate, healthcare-associated infections, bacterial colonization 


\section{Introduction}

Healthcare-associated infections (HAI), including those originating in hospitals, are currently one of the most important challenges for modern medicine. They are a common cause of morbidity and mortality, and result in adverse effects. Healthcare-associated infections are responsible mainly for pneumonia, as well as bloodstream, urinary tract and surgical site infections. The estimated number of HAI in American hospitals in 2002 was approx. 1.7 million. ${ }^{1}$ Especially dangerous are infections with antibiotic-resistant (AR) bacteria. It has been proven that such infections affect disability-adjusted life-years (DALYs) ${ }^{1-3}$ They are also associated with prolonged hospital stays and lengthy treatment with antibiotics or steroids. ${ }^{4}$

Besides the serious consequences to patients, HAI are also dangerous to healthcare professionals and generate significant costs to the healthcare system. It was estimated that in 2007 (after adjusting from previous data), the cost resulting from HAI treatment in American hospitals exceeded 33 billion dollars, and the benefits from prevention exceeded 6 billion dollars. ${ }^{5}$ Therefore, recently, great emphasis is being placed on HAI and special preventive procedures such as enhanced active surveillance, patient isolation, hand hygiene, surface disinfection, and enhanced education of patients and healthcare professionals are being implemented in healthcare institutions. Despite these procedures, more invasive medical technologies and the demographics of society will increase the chances of HAI.

The risk factors for HAI are predominantly previous hospitalization, urinary tract catheterization, terminal illness, and broad-spectrum antibiotic therapy ${ }^{6,7}$ The challenge for medical personnel is to prevent and minimize the occurrence of healthcare infections, which are partly unavoidable. Healthcare-associated infections has become a patient safety problem and a public health problem. ${ }^{1}$ Patients with infectious diseases display specific clinical symptoms, while carriers usually are admitted to hospitals due to other clinical conditions. Therefore it is of great importance to detect carriers of bacteria at admission and isolate them in single-patient rooms to prevent further spread of infection. ${ }^{4,8}$ International recommendations prefer active screening rather than passive; however, these strategies depend mainly on respective hospital recommendations. ${ }^{9}$

Properly diagnosed carriers could be decolonized. It has been proven that decolonization reduces the risk of surgical site infection and the rate of infections in intensive care units. In the case of methicillin-resistance Staphylococcus aureus (MRSA), decolonization with chlorhexidine and mupirocin lowers the risk of infection by $30 \%$ compared to education regarding decolonization alone.

The aim of this study was to evaluate the occurrence rate of colonization with various bacteria among patients admitted to the Department of Immunology, Transplantology and Internal Diseases (ITID).
We focused on the quantitative rather than qualitative aspect of the problem. In each case, a detailed microbiological diagnosis was performed, including identification of antibiotic resistance, and appropriate medical therapy was implemented; however, this data was not considered in this study.

\section{Material and methods}

The study protocol was approved by the Bioethics Committee of the Medical University of Warsaw (approval No. AKKB/123/2019). The study used retrospective analysis of data regarding patients admitted into the ITID, Infant Jesus Teaching Hospital, Medical University of Warsaw, Poland, between November 2015 and May 2017. Data regarding the number of hospitalizations since enrollment into the study was collected in January 2019.

Patients enrolled in the study $(\mathrm{n}=293)$ had been admitted into the ITID for the first time or with no history of hospitalization during the previous year other than hospitalization immediately prior to the admission. Patients had been admitted to the ITID from another hospital, another ward of the same hospital or from home. None of them had clinical signs of infection. Patients for oneday hospitalization were excluded from the study. Because of missing data, the investigated group consisted of 280 patients. For each participant, data collected included sex, age at admission, data regarding previous location, and medical history of transplantation or other non-communicable diseases. The characteristics of the investigated group are included in Table 1. In all patients, nasal and rectal swabs were collected, and stool and urine samples were obtained on the day of admission. They were examined for carriage of the following pathogens: vancomycin-resistant Enterococci (VRE), extended spectrum beta-lactamases (ESBL) producing Enterobacteriaceae as well as carbapenemase producing Enterobacteriaceae (CPE). Moreover, patients were examined for the presence of Clostridium difficile (stool samples only). A patient was recognized as colonized if any of the above bacteria were found. In connection with usual multiresistance, ESBL- or/and carbapenemase-producing Escherichia coli in this work were called antibioticresistant E. coli (AR E. coli), and antibiotic-resistant Klebsiella pneumoniae (AR K. pneumoniae) was named similarly.

All microbiological procedures were performed at the Chair and Department of Medical Microbiology of the Infant Jesus Teaching Hospital. The various specimens from the patients were cultured according to standard microbiological procedures, depending on the kind of specimen and the aim of examination (infection/colonization). In all cases, the appropriate bioMerieux (Marcyl'Étoile, France) media were used. Isolates were identified using a mass spectrometer (Vitek MS; bioMerieux). Susceptibility testing was performed using the Vitek 2 or disc diffusion method and the results were interpreted 
Table 1. Demographic and clinical details of the investigated group

\begin{tabular}{|c|c|c|c|}
\hline Factor & Description & $n$ & $\%$ of $n=280$ \\
\hline \multirow{2}{*}{ Sex } & male & 142 & $50.7 \%$ \\
\hline & female & 137 & $49.3 \%$ \\
\hline \multirow{3}{*}{ Admission group } & home & 173 & $61.8 \%$ \\
\hline & other hospital & 79 & $28.2 \%$ \\
\hline & another ward in the same hospital & 28 & $10.0 \%$ \\
\hline \multirow{4}{*}{ Medical history } & kidney transplantation (KTx) & 145 & $51.8 \%$ \\
\hline & liver transplantation (LTx) & 64 & $22.9 \%$ \\
\hline & heart transplantation (Htx) & 2 & $0.7 \%$ \\
\hline & other non-communicable disease (INT) & 71 & $25.4 \%$ \\
\hline Factor & Mean (SD) & Min-Max & Median \\
\hline Age & $50.19 \pm 15.02$ & $37-61$ & 51 \\
\hline
\end{tabular}

according to European Committee on Antimicrobial Susceptibility Testing (EUCAST) recommendations. ${ }^{11}$ For detection of carbapenemase production, the combination disk test was performed and results were confirmed using biochemical CarbaNP test. ${ }^{12,13}$ Production of extended spectrum beta-lactamases (ESBLs) was examined with modified double disc synergy test (DDST). ${ }^{14}$ All tests were recommended by EUCAST. ${ }^{15}$

\section{Statistical analysis}

Statistical analysis used R software v. 3.5.0 (www.r-project. org). Descriptive statistics of the data were generated using standard statistical parameters: percentage, mean and standard deviation (SD), median, and minimum and maximum (min and max). Median values in the 2 independent groups were compared using the nonparametric Mann-Whitney U test. The correlations between pairs of numerical parameters were studied using $X^{2}$ test or Fisher's test. Multivariate analysis was conducted using a logistic regression model based on Wald's statistics. In all analyses, results were considered significant if the $\mathrm{p}$-value was $<0.05$.

\section{Results}

From the investigated group, 191 of patients were colonized with AR bacteria (men, $\mathrm{n}=93$, women, $\mathrm{n}=98$ ), which comprised $68.2 \%$ (accordingly, 48.7\% of men and 51.3\% of women) of all study population. The incidences of colonization were not influenced by age or sex. From the colonized group, $58.6 \%$ were admitted from home $(\mathrm{n}=112)$, $32.5 \%$ were admitted from another hospital $(n=62)$ and the rest was admitted from another ward of the hospital $(8.9 \%, \mathrm{n}=17)$. Among the colonized patients, $57.6 \%$ ( $\mathrm{n}=110$ ) had a history of kidney transplantation, $19.4 \%$ $(n=37)$ had a history of liver transplantation, $1 \%(n=2)$ were after heart transplantation, and $23 \%(n=44)$ were admitted to the ward due to other non-communicable diseases. The $\chi^{2}$ test revealed a significant correlation between kidney transplantation and colonization, regardless of the localization of colonization ( $\mathrm{p}=0.0065)$.

According to the results of the logistic regression analysis, the risk of colonization was associated with admission from another hospital and a history of kidney transplantation ( $\mathrm{p}=0.0136$ and $\mathrm{p}<0.001$, respectively). The risk of colonization among patients admitted from another hospital was almost 2.2 times higher compared to the group of patients who were admitted from home (odds ratio $(\mathrm{OR})=2.151,95 \%$ confidence interval $(95 \% \mathrm{CI})=1.139-$ 5.062), and were almost 2.4 times higher among patients after kidney transplantation compared to other patients $(\mathrm{OR}=2.353,95 \% \mathrm{CI}=1.383-4.003)$.

The most common type of pathogen among the investigated group was vancomycin-resistant Enterococci spp. (VRE) (24.9\%). Less common were AR E. coli $(21.5 \%)$ and AR K. pneumoniae (15.4\%). Clostridium difficile was diagnosed in $14.3 \%$ of patients. A similar panel of pathogens was observed in the group of patients with different medical history. None of the investigated parameters (age, sex, medical history, and the place of previous location) influenced the incidence of particular pathogens colonization, excluding AR E. coli and VRE. Women were infected by AR E. coli more often than men $(\mathrm{p}=0.022)$. Infection with VRE was less common among patients with non-communicable diseases compared to the rest of the investigated group $(p=0.047)$. The Fisher's test revealed that more patients free of VRE colonization were admitted from home than from another hospital ( $\mathrm{p}=0.0013$ ). The particular pathogen colonization in the group of patients after heart transplantation were not considered due to the small number of such participants.

Forty patients were colonized with $C$. difficile, which comprised $14.3 \%$ of the whole investigated group. From this group, 23 patients (57.5\%) were admitted to the ITID from home, 13 patients were admitted from another hospital (32.5\%) and 4 patients were admitted from another ward of the hospital (10\%). 


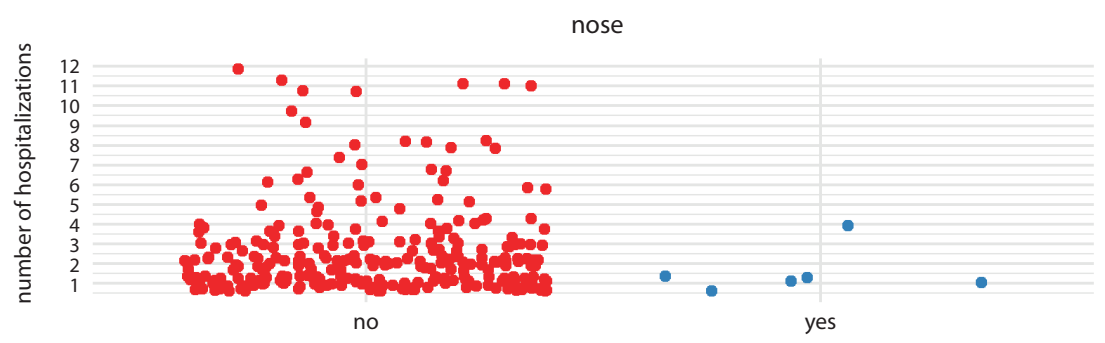

Fig. 1. The number of hospitalizations during the whole observational period of patients belonging to the whole investigated group, divided into

- no subgroups: those who had a positive

- yes culture of rectum swabs $(n=110)$, feces samples (C. difficile, $n=40$ ), urine samples $(n=97)$ and nasal swabs $(n=6)$, and according to the number of positive cultures obtained in each patient $(1,2$ or 3$)$
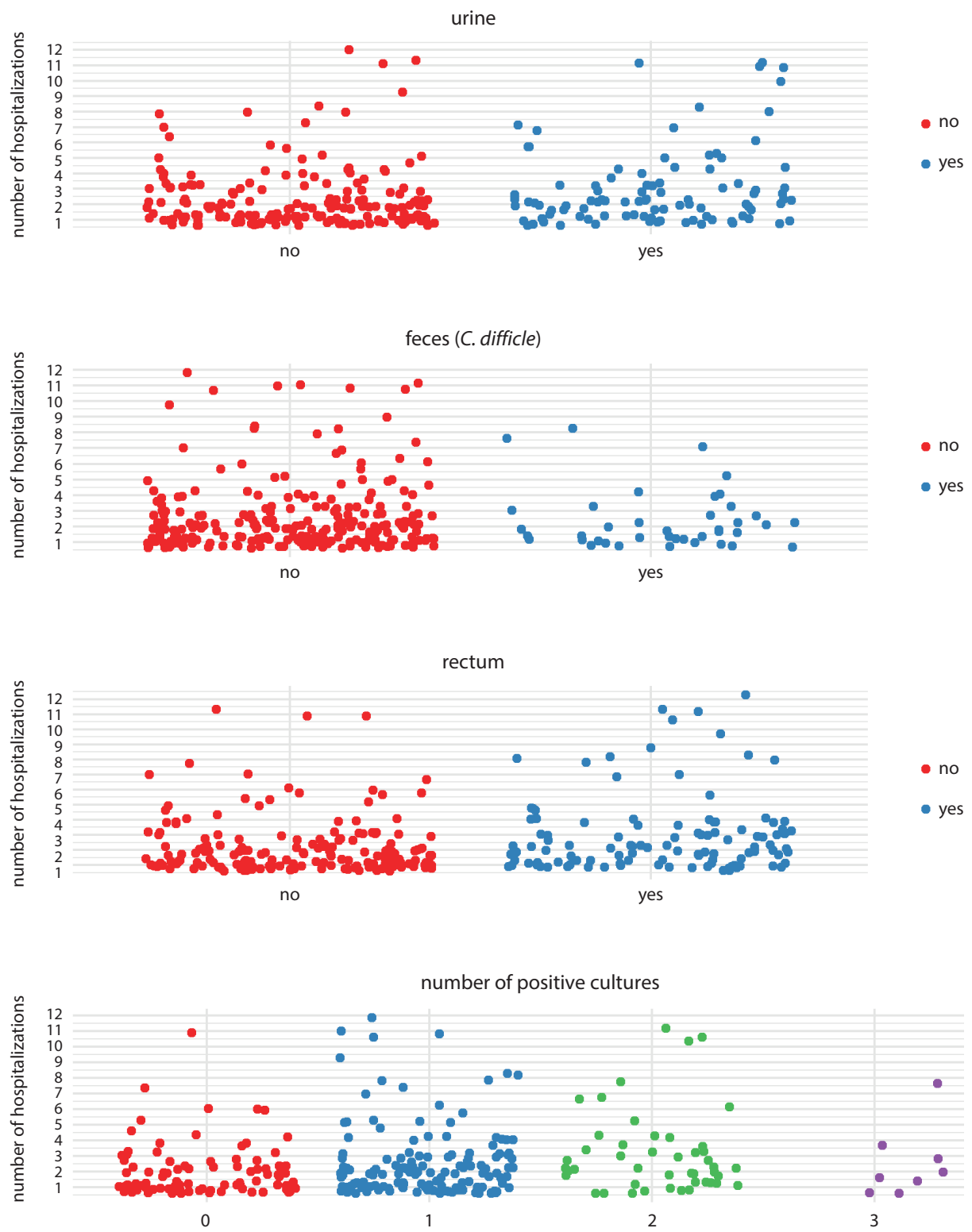

The number of hospitalizations during the whole observational period was higher in the group of colonized patients compared to non-colonized in the whole investigated group $(2.76 \pm 2.4$ vs $2.07 \pm 1.68, \mathrm{p}=0.0099)$. The same was observed in the group of patients with positive urine culture in comparison to those with negative urine culture ( $\mathrm{n}=97,2.95 \pm 2.5 \mathrm{vs} \mathrm{n}=183,2.33 \pm 2.02, \mathrm{p}=0.0012$ ), and in the group of patients with positive rectal swab in comparison to those with negative rectal swab $(n=110,3.0 \pm 2.57$ vs $\mathrm{n}=170,2.25 \pm 1.9, \mathrm{p}=0.0044)$. Clostridium difficile colonization did not influence the number of hospitalizations. The influence of positive nasal swabs on the number of hospitalizations was not considered due to the small number of such patients. The number of hospitalizations correlated positively with the number of positive cultures obtained from the same patients; however, the correlation was weak (Spearman's rho $=0.18, \mathrm{p}=0.0274)$. The results described above shown in Fig. 1. 


\section{Discussion}

Our results showed that almost $70 \%$ of patients free of any clinical symptoms of infection at admission to the hospital were colonized by various AR bacteria. This is very high rate but similar results had already been reported. ${ }^{6,7}$ However, other studies reported much lower rates. ${ }^{4}$ The high rate of colonization in our patients could result from their medical history. The majority of them had undergone organ transplantation and were under chronic immunosuppression treatment. We showed that renal transplantation increased the risk for colonization almost 2.5 times. Colonized patients could be a potential source of infection; however, the transmission rate depends on the epidemiology of the pathogens. ${ }^{8,16}$ The most frequently diagnosed pathogen in our study was VRE. According to literature data, the frequency of particular pathogens' colonization and infection rates differ depending on the specificity of the healthcare facility. ${ }^{17}$ In hematopoietic stem cell transplant recipients, Staphylococci spp. and Enterobacteriaceae were the most frequent pathogens causing infections, and in the oncologic ward, Enterococci spp. were detected in more than $40 \%$ of patients. ${ }^{4,18}$ The epidemiology of pathogens do not vary significantly with regard to age, sex or medical history. Similar results were obtained by Chmielarczyk et al. ${ }^{19}$ Special attention should be placed on C. difficile colonization. Clostridium difficile is a Gram-positive, spore-forming anaerobic bacteria, and it is recognized as one of the most important pathogens in healthcare settings as it is the major cause of antibiotic therapy associated infections infections and could contribute to increased mortality. ${ }^{19,20}$ It is distinct from other microorganisms due to the fact that its spores can persist in the environment for a long time. Asymptomatic colonization of $C$. difficile is not clearly defined; however, it is recognized in the absence of infection symptoms. ${ }^{21}$ The number of colonized patients is higher than symptomatic, ${ }^{22}$ but it varies between different patient groups. In a healthy population, colonization with these bacteria varied between $0 \%$ and $15 \%$, while in hospitalized patients, it reaches $30 \%$, which is more or less in line with our findings, because in our group more than $14 \%$ of patients were colonized at admission to the hospital. ${ }^{21}$ In the case of $C$. difficile, transmission from person to person usually takes place, but colonized asymptomatic patients have the potential to contaminate the environment and subsequently other patients. ${ }^{23}$

In this study we focused on quantitative analysis of the AR bacteria colonization rate among patients of ITID. We found that colonized patients are most frequently admitted from other hospitals. This is in line with previously recognized risk factors for colonization, e.g., hospitalization within 2 months prior to current hospitalization ${ }^{22}$; however, it is worth noting that the majority of colonized patients in our study were admitted to the ward from their homes.
Therefore, we recommend also treating such patients as potentially colonized and a potential source of infection. However, we should note that patients after organ transplantation usually had a history of many previous hospitalizations despite the fact that in this study they were not hospitalized in 1 year prior to the study. Another important finding is that colonization is a risk factor for an increase in the number of hospitalizations over the 2.5-year observational period, especially in the case of urinary tract and gastrointestinal tract colonization. Surprisingly, colonization with C. difficile did not increase the number of hospitalizations. Similar results were obtained by Huang et al. Patients who underwent decolonization of MRSA infection had lower risk of hospitalization over 1 year after discharge. ${ }^{10}$

Interventions to reduce transmission depend on the epidemiology of the bacteria and the main route of acquisition. It was shown that identification of carriers and implementation of preemptive isolation, and contact precautions had little effectiveness on transmission of, for instance, MRSA, VRE or HRE (highly resistant Enterobacteriaceae). Hand hygiene is effective in protection from MRSA and VRE transmission, while the data regarding the effectiveness of chlorhexidine bathing is conflicting. It should be noted that MRSA and VRE can also colonize the skin and environment. ${ }^{8}$ Therefore, other methods should be considered to protect everyone from bacterial transmission, such as decolonization. ${ }^{8}$ Also, current guidelines do not recommend active screening for $C$. difficile colonized patients because its impact on infection transmission prevention is low. ${ }^{21}$ The reason is that there is limited information on the duration of the latent period in the case of contact with $C$. difficile; therefore, patients exposed but not yet colonized could not be detected. ${ }^{24}$ Another reason is that the treatment for colonized asymptomatic patients is not recommended. Nevertheless, intensive infection control practices like the use of gloves and enhanced environmental cleaning should be implemented with regard to the high number of patients potentially colonized with C. difficile. ${ }^{21,23}$

\section{Conclusions}

The rate of colonization at admission to the ward could be high, depending on previous hospitalization and medical history. The rate of colonization in patients admitted to the ward from home is also high enough to treat them as potentially colonized. The epidemiology of the pathogens do not vary significantly with regard to age, sex, medical history, or location directly prior to current hospitalization, excluding AR E. coli, which was more often in urine samples from women, and VRE, which were less common among patients with non-communicable diseases and admitted from home. Colonization significantly increased the post-discharge hospitalization rate. 


\section{ORCID iDs}

Bożena Czarkowska-Pączek (10) https://orcid.org/0000-0002-1023-3057 Elżbieta Wawiórko (1) https://orcid.org/0000-0001-5634-4644 Grażyna Młynarczyk @i) https://orcid.org/0000-0002-6360-2688 Leszek Paczek (1) https://orcid.org/0000-0003-0160-3009

\section{References}

1. Klevens RM, Edwards JR, Richards CL Jr, et al. Estimating health careassociated infections and deaths in U.S. hospitals, 2002. Public Health Rep. 2007;122(2):160-166.

2. Friedrich AW. Control of hospital acquired infections and antimicrobial resistance in Europe: The way to go. Wien Med Wochenschr. 2019;169(Suppl 1):25-30. doi:10.1007/s10354-018-0676-5

3. Cassini A, Hogberg LD, Plachouras D, et al. Attributable deaths and disability-adjusted life-years caused by infections with antibioticresistant bacteria in the EU and the European Economic Area in 2015: A population-level modelling analysis. Lancet Infect Dis. 2019;19(1): 56-66. doi:10.1016/S1473-3099(18)30605-4

4. Kampmeier S, Knaack D, Kossow A, et al. A. Weekly screening supports terminating nosocomial transmission of vancomycin-resistant Enterococci on an oncologic ward: A retrospective analysis. Antimicrob Resist Infect Control. 2017;6:48. doi:10.1186/s13756-017-0206-z

5. Scott II RD. The direct medical costs of health-care associated infections in U.S. hospitals and the benefits from prevention. https://www. cdc.gov/hai/pdfs/hai/scott_costpaper.pdf. Accessed February 4, 2019.

6. Ludden C, Cormican M, Vellinga A, Johnson JR, Austin B, Morris D. Colonisation with ESBL-producing and carbapenemase-producing Enterobacteriaceae, vancomycin-resistance enterococci, and methicillin-resistant Staphylococcus aureus in a long-term care facilities. BMC Infect Dis. 2015;15:168.

7. Thuy DB, Campbell J, Nhat LTH, et al. Hospital-acquired colonization and infections in Vietnamese intensive care unit. PLoS One. 2018; 13(9):e0203600. doi:10.1371/journal.pone.0203600

8. Derde LPG, Cooper BS, Goossens H, et al. Interventions to reduce colonisation and transmission of antimicrobial-resistant bacteria in intensive care units: An interrupted time series study and cluster randomised trial. Lancet Infect Dis. 2014;14(1):31-39. doi:10.1016/S14733099(13)70295-0

9. Faron ML, Ledeboer NA, Buchan BW. Resistance mechanisms, epidemiology, and approaches to screening for vancomycin-resistant Enterococcus in the health care setting. J Clin Microbiol. 2016;54(10): 2436-2447. doi:10.1128/JCM.00211-16
10. Huang SS, Singh R, McKinnell JA, et al. Decolonization to reduce postdischarge infection risk among MRSA carriers. N Engl J Med. 2019; 380(7):638-650. doi:10.1056/NEJMoa1716771

11. The European Committee on Antimicrobial Susceptibility Testing. Breakpoint tables for interpretation of MICs and zone diameters, v. 9.0, 2019. http://www.eucast.org/clinical_breakpoints/.

12. Miriagou V, Tzelepi E, Kotsakis SD, Daikos GL, Bou Casals J, Tzouvelekis LS. Combined disc methods for the detection of KPC- and/or VIM-positive Klebsiella pneumoniae: Improving reliability for the double carbapenemase producers. Clin Microbiol Infect. 2013;19(9): E412-415.

13. Nordmann P, Poirel L, Dortet L. Rapid detection of carbapenemaseproducing Enterobacteriaceae. Emerg Infect Dis. 2012;18(9):1503-1507.

14. Paterson DL, Bonomo RA. Extended-spectrum $\beta$-lactamases: A clinical update. Clin Microbiol Rev. 2005;18(4):657-686.

15. The European Committee on Antimicrobial Susceptibility Testing. The EUCAST guideline on detection of resistance mechanisms v. 1.0 (2013-12-11). http://www.eucast.org/resistance_mechanisms/.

16. Sekowska A, Gospodarek E, Kusza K. The prevalence of infections and colonisations with Klebsiella pneumoniae strains isolated in ICU patients. Anaesthesiol Intensive Ther. 2014;46(4):295-298.

17. Talaga K, Odrowąż-Konduracka D, Paradowska B, et al. Typing of Enterococcus spp. strains in 4 hospitals in the Małopolska region in Poland. Adv Clin Exp Med. 2018;27(1):111-117. doi:10.17219/acem/68265

18. Balletto $E$, Mikulska M. Bacterial infections in hematopoietic stem cell transplant recipients. Mediterr J Hematol Infect Dis. 2015;7(1):e2015045. doi:10.4084/MJHID.2015.045

19. Chmielarczyk A, Pobiega M, Ziółkowski G, et al. Severe infections caused by multidrug-resistant non-fermentative bacilli in southern Poland. Adv Clin ExpMed. 2018;27(3):401-407. doi:10.17219/acem/68545

20. Rodriguez C, Taminiau B, Korsak N, et al. Longitudinal survey of Clostridium difficile presence and gut microbiota composition in a Belgian nursing home. BMC Microbiol. 2016;16(1):229.

21. Furuya-Kanamori L, Marquess J, Yakob L, et al. Asymptomatic Clostridium difficile colonization: Epidemiology and clinical implications. BMC Infect Dis. 2015;15:516. doi:10.1186/s12879-015-1258-4

22. Loo VG, Bourgault AM, Poirier L, et al. Host and pathogen factors for Clostridium difficile infection and colonization. N Engl J Med. 2011; 365(18):1693-1703. doi:10.1056/NEJMoa1012413

23. Curry SR, Muto CA, Schlackman JL, et al. Use of multilocus variable number of tandem repeats analysis genotyping to determine the role of asymptomatic carriers in Clostridium difficile transmission. Clin Infect Dis. 2013;57(8):1094-1102. doi:10.1093/cid/cit475

24. Yakob L, Riley TV, Paterson DL, Clements AC. Clostridium difficile exposure as an insidious source of infection in healthcare settings: An epidemiological model. BMC Infect Dis. 2013;13:376. doi:10.1186/14712334-13-376 